\title{
Intraconal Metastasis Leading to Diagnosis of Hepatocellular Carcinoma
}

\author{
Irappa Madabhavi 1,*, Sandeep KS ${ }^{2}$, Rahul Dharmarajan Lethika ${ }^{2}$, Satish Tumbal ${ }^{3}$ \\ Arun T Miskin ${ }^{4}$, Malay Sarkar ${ }^{5}$, Mitul Modi ${ }^{6}$
}

1. Department of Medical and Pediatric Oncology and Hematology, Kerudi Cancer Hospital, Bagalkot, Karnataka, India

2. Department of Radiation Oncology, Kerudi Cancer Hospital, Bagalkot, Karnataka, India

3. Department of Radiology, Kerudi Cancer Hospital, Bagalkot, Karnataka, India

4. Department of Pathology, SNMCMedical College, Bagalkot, Karnataka, India

5. Department of Pulmonary Medicine, Indira Gandhi Medical College, Shimla, Himachal Pradesh, India

6. Department of Pathology, University of Pennsylvania, Philadelphia, PA, USA

\section{* Corresponding Author:}

Dr. Irappa Madabhavi, MBBS, MD, DM, ECMO

Kerudi Cancer Hospital, Baagalkot, Karnataka, India-587101

Telefax: + 917619501323

Email: irappamadabhavi@gmail.com

Received: 18 Mar. 2019

Accepted: 03 Dec. 2019

\section{ABSTRACT}

Hepatocellular carcinoma (HCC) is the commontumor of the liver and the third most common cause of cancer-related mortality worldwide. Patients with HCC may have metastasis to different sites. Intrahepatic and extrahepatic metastases are found in ( $50-75 \%)$. Lung and regional lymph nodes are the most commonly involved sites. Metastasis to bone, skin, and adrenal glands are rare. Orbit metastasis and intracranial invasion are extremely rare. We are presenting a case of HCC that metastasized to the orbital cavity. The patient presented with progressive proptosis of the eyeball with retrobulbar and intracranial invasion and involvement of the sub-scalp region. Based on the imaging findings, it was initially misdiagnosed as meningioma; however, histopathological examination of the biopsy specimen resulted in a definitive diagnosis of HCC metastasis. The present case reveals that the alternative diagnosis of metastasis must be considered when diagnosing retrobulbar lesions in patients with HCC.

\section{KEYWORDS:}

Hepatocellular carcinoma, Intraconal metastasis, Sorafenib, Imaging

\section{Please cite this paper as:}

Madabhavi I, Sandeep KS S, Lethika Dharmarajan R, TumbalS, T MiskinA, Sarkar M, Modi M. Intraconal Metastasis Leading to Diagnosis of Hepatocellular Carcinoma. Middle East $J$ Dig Dis 2020;12:48-51. doi: 10.15171/mejdd.2020.164.

\section{INTRODUCTION}

Metastasis to the orbital cavity is an unusual event and believed to occur in approximately $2-3 \%$ of patients with systemic cancer. The incidence of metastasis to the orbital cavity varies from $1 \%$ to $13 \%$ of orbital tumors. ${ }^{1,2}$ Patients who have small orbital lesions may remain asymptomatic. Poor general condition in patients with the widespread disease may mask the orbital symptoms leading to a low rate of referral to concerned specialists. ${ }^{3}$ The actual incidence of orbital metastasis is thought to be higher than that described in the literature and this discrepancy is because of improved treatment leading to an increase in the median survival of patients with cancer, which in turn increases the chances of development of metastatic lesions the orbit. Advances in the diagnostic methods led to the increased detection of lesions and an increased volume of medical literature on orbital metastasis has increased the awareness of these lesions. ${ }^{4}$ 


\section{CASE REPORT}

72-year-old male patient, with ECOG (Eastern Cooperative Oncology Group) performance status of 1 , presented with progressive proptosis of the left eye with pain, weight loss, loss of appetite, and weakness since 3 months earlier. There was no history of trauma to the left eye, headache, vomiting, abnormal body movements, fever, and congestion or discharge from the eye. He was non-alcoholic, non-smoker, and non-diabetic. The patient had no history suggestive of any drug intake or alcohol consumption or jaundice in the past or any immunecompromised status and his family history was not contributory. On admission, his vitalsigns and temperature were within the normal range for his age. On examination left eye was proptosed with a mass in the upper, outer, and lateral aspect of the orbit, which was soft to firm in consistency, mild to moderately-tender, not fixed to the underlying tissue and not associated with any discharge from the local site. The size of the mass was around $5.5 \mathrm{~cm} \times 3.50$ $\mathrm{cm}$ (figure. 1). On per abdominal examination, the liver margin was palpated $5 \mathrm{~cm}$ below the right costal margin in mid-clavicular line and there was no ascites.

Complete blood count and renal functions were within normal limits. His liver function tests such asserum alanine aminotransferase (ALT), serum aspartate transaminase (AST), serum gammaglutamyltranspeptidase (GGT), total protein, albumin level, lactate dehydrogenase (LDH), ferritin, and prothrombin time (PT) levels were within the normal range. Serum alpha-fetoprotein [AFP] was $1.69 \mathrm{ng} / \mathrm{mL}$. The patient's serology was positive for hepatitis B surface antigen and hepatitis B virus-DNA. His serum was negative for hepatitis $\mathrm{C}$ virus (anti-HCV) and PCR for HCV-RNA for hepatitis C, anti-mitochondrial antibody and carcinoembryonic antigen (CEA). Computed tomography (CT) of head and neck revealed heterogeneously enhancing irregular extraconal mass lesion measuring approximately $5.7 \times 4.7 \mathrm{~cm}$ in the superolateral aspect of the left orbit. It was breaching the lateral wall and roof of the orbit and extending into subcutaneous scalp tissue layers of the left frontal region. It was causing mass effect on eyeball resulting in the proptosis of the left eyeball and invading the left-sided extraconal muscles, left lateral rectus and superior oblique muscles (figure 2). Triphasic abdominal CT showed hepatomegaly withmultiple heterogeneously enhancing lesions in both lobes of the liver, the largest being $83.8 \times 84.6 \mathrm{~mm}$ (figure 3 ). Cytology smears of fine needle aspiration from proptotic lesion and liver mass showed clusters and sheets of round to polyhedral cells, which were scattered individually with enlarged, hyperchromatic nuclei, prominent nucleoli, and moderately abundant eosinophilic cytoplasm. Anisonucleosis was also seen. Some cells showed intranuclear inclusions. Many atypical stripped nuclei were seen. Scattered cells with large, bizarre nuclei were also seen (figure 4). On further examination tumor cells had strong immunoreactivity for hepatocyte paraffin 1 (HepPar 1) and CD 10 but negative for TTF-1 (thyroid transcription factor 1), CEA (carcinoembryonic antigen), ALK-1(Activin Receptor- like Kinase), LCA(Leukocyte common antigen), vimentin, S-100, and chromogranin. The results of immunohistochemistry were thus consistent with a diagnosis of metastatic hepatocellular carcinoma (HCC).

Sorafenib $400 \mathrm{mg}$ twice a day was started for the patient and he is under treatment until this date. After one month of starting the sorafenib there was a significant improvement in the performance status and symptoms in the form of increased appetite, increased weight, and subjective wellbeing of the patient. There was also a decrease in the size of the liver mass and the orbital mass. Imaging studies were suggestive of a decrease in the size of the intraorbitaland liver masses. The patient is under regular evaluation in our center for clinical signs and signs of liver decompensation. Imaging studies with two monthly dynamic CT of the abdomen is done for any progression of the disease since 7 months age.

\section{DISCUSSION}

Worldwide HCC is the seventh most common cancer and around 626,000 new patients are diagnosed globally each year; among them, $50 \%$ are in China, with a mortality rate of $94 \% .^{5} \mathrm{HCC}$ is associated with alcoholic liver diseases, infections like hepatitis $\mathrm{C}$ virus, and hepatitis 


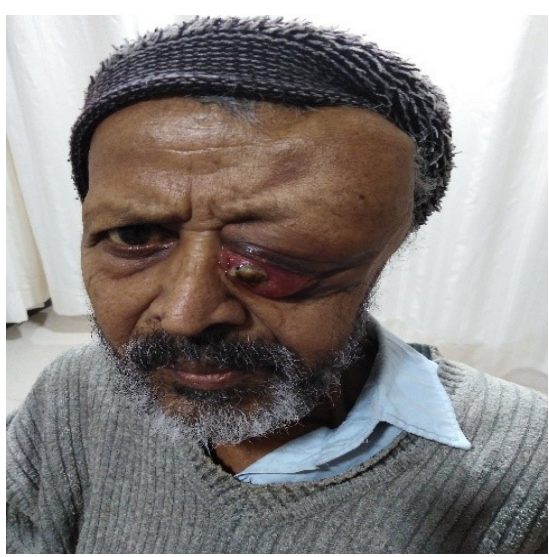

Fig.1: Proptotic left eye with a mass of size $5.5 \times 3.5 \mathrm{~cm}$ in the upper, outer, and lateral side of the left eye is shown.

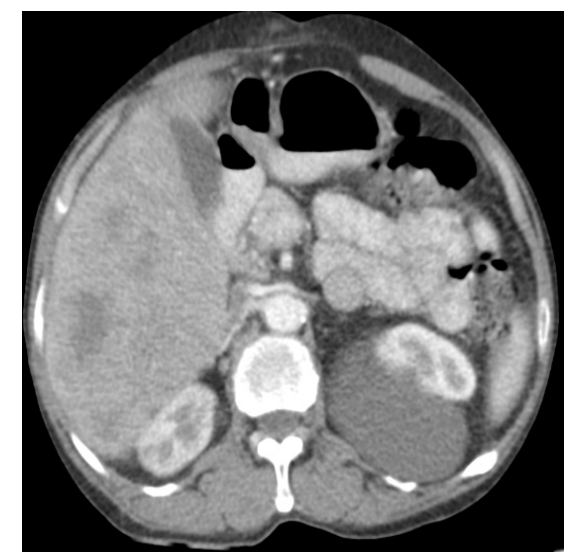

Fig.3: Triphasic abdominal tomogram shows hepatomegaly measuring about $17 \mathrm{~cm}$ in span and multiple heterogeneous enhancing lesions in both lobes of the liver, the largest measuring $83.8 \times 84.6 \mathrm{~mm}$.

B virus, autoimmune hepatitis, steatohepatitis, primary biliary and sclerosing cholangitis, intake of aflatoxincontaminated food, diabetes, and obesity. ${ }^{6}$

One study from Australia showed metastasis to the orbit and malignant melanoma (20\%) followed by prostatic carcinoma (13\%), while in another study done in the People's Republic of China, the most common primary cancers that metastasized to the orbit in the descending order were nasopharyngeal carcinoma (30.3\%), lung cancer (8.7\%), liver cancer $(6.5 \%)$, and breast cancer $(4.35 \%) .{ }^{7,8}$

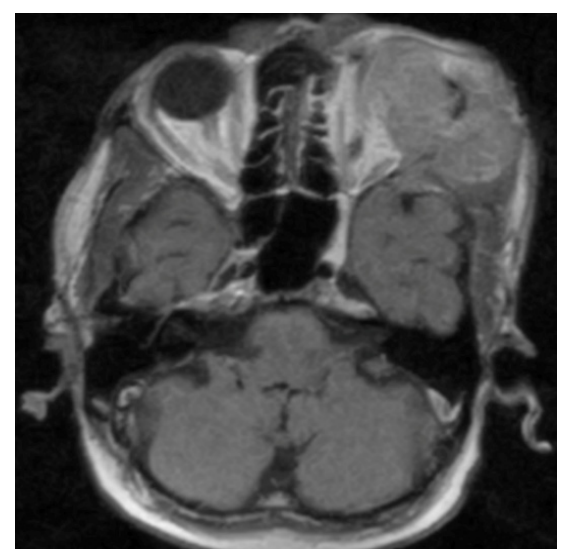

Fig.2: Computed tomography of head and neck revealing heterogeneously enhancing irregular extraconal mass lesion measuring approximately $5.7 \times 4.7 \mathrm{~cm}$ in the superolateral aspect of the left orbit. It is breaching the lateral wall and roof of the orbit and extending into subcutaneous scalp tissue layers of the left frontal region. It is causing mass effect on eyeball resulting in the proptosis of the left eyeball and invading left-sided extraconal muscles, left lateral rectus, and superior oblique muscles.

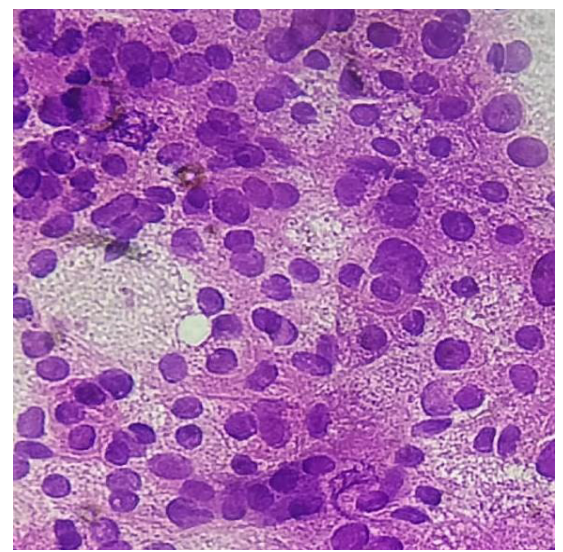

Fig.4: Fine needle aspiration cytology smears from proptotic lesion and liver massshow individually scattered, clusters, and sheets of round to polyhedral cells with enlarged, hyperchromatic nuclei, prominent nucleoli, and moderately abundant eosinophilic cytoplasm. Anisonucleosis is also seen. Some cells show intranuclear inclusions. Many atypical stripped nuclei are seen. Scattered cells with large, bizarre nuclei are seen.

Oncologists should have knowledge regarding, primary neoplasms and metastatic tumors to the orbit, which causes proptosis and pain. Primary orbital tumors associated with painful proptosis are adenoid cystic carcinoma of the lacrimal gland, malignant peripheral nerve sheath tumor (malignant schwannoma), and direct orbital invasion by neurotrophic squamous cell carcinoma from the skin of the eyelid and periorbital region. Among the metastatic lesions to the orbit, one must include prostatic adenocarcinoma, HCC, breast cancer, Langerhans cell histiocytosis, 
and lung carcinoma.

HCC is a type of tumor with a rich blood supply. Vascular endothelial growth factor (VEGF), as the most important vascular growth factor, is significantly overexpressed in patients with HCC compared with patients with cirrhosis, hepatitis, and so forth. For patients with and without bone metastasis, a statistical difference is also observed in the expression level of VEGF. This factor may facilitate the formation of a metastatic niche and subsequent mass. ${ }^{9}$

Goldberg and Rootman described the features of orbital metastasis as ${ }^{4,10}$ :

1. Infiltrative with prominent motility restriction and sometimes enophthalmos (most common);

2. Mass effect with proptosis and/or globe displacement;

3. Inflammatory; and

4. Functional.

CT and magnetic resonance imaging (MRI) are the principal means for evaluating orbital lesions. The definitive diagnosis of an orbital metastasis requires tissue evaluation by a pathologist. Fine needle aspiration has been advocated as a good diagnostic modality. ${ }^{11}$

The preferred treatment of metastatic or inoperable HCC includes sorafenib and lenvatinib. Other subsequent lines of treatment if the disease progresses on preferred treatments include regorafenib, cabozantinib, ramucirumab (if AFP > $400 \mathrm{ng} / \mathrm{ml}$ only), sorafenib after first-line lenvatinib, and immunotherapeutic agents such as nivolumab and pembrolizumab.

\section{ETHICAL APPROVAL}

There is nothing to be declared.

\section{CONFLICT OF INTEREST}

The authors declare no conflict of interest related to this work.

\section{REFERENCES}

1. Albert DM, Rubenstein RA, Scheie HG. Tumor metastasis to the eye. I. Incidence in 213 adult patients with generalized malignancy. Am J Ophthalmol 1967;63:727-32. doi:10.1016/0002-9394(67)91298-6.

2. Bloch RS, Gartner S. The incidence of ocular metastatic carcinoma. Arch Ophthalmol 1971;85:6735. doi:10.1001/archopht.1971.00990050675005.

3. Ahmad SM, Esmaeli B. Metastatic tumors of the orbit and ocular adnexa. Curr Opin Ophthalmol 2007;18:40513.doi:10.1097/ICU.0b013e3282c5077c.

4. Goldberg RA, Rootman J, Cline RA. Tumors metastatic to the orbit: a changing picture. Surv Ophthalmol 1990;35:1-24. doi:10.1016/00396257(90)90045-w.

5. Parkin DM, Bray F, Ferlay J, Pisani P. Global cancer statistics, 2002. CA Cancer J Clin 2005; 55:74-108. doi:10.3322/canjclin.55.2.74.

6. Madabhavi I, Patel A, Choudhary M, Anand A, Panchal H, Parikh S. Right cervical lymphadenopathy: a rare presentation of metastatic hepatocellular carcinoma. Gastroenterol Hepatol Bed Bench 2014;7:177-82.

7. Valenzuela AA, Archibald CW, Fleming B, Ong L, O'Donnell B, Crompton J J,et al. Orbital metastasis: clinical features, management and outcome. Orbit 2009;28:153-9. doi: 10.1080/01676830902897470.

8. Yan J, Gao S. Metastatic orbital tumors in southern China during an 18-year period. Graefes Arch ClinExp Ophthalmol 2011;249:1387-93. doi: 10.1007/s00417011-1660-6.

9. Kaseb AO, Hanbali A, Cotant M, Hassan MM, Wollner I, Philip PA. Vascular endothelial growth factor in the management of hepatocellular carcinoma: a review of literature. Cancer 2009;115:4895-906. doi: 10.1002/cncr.24537.

10. Goldberg R, Rootman J. Clinical characteristics of metastatic orbital tumors. Ophthalmology 1990;97:6204. doi:10.1016/s0161-6420(90)32534-4.

11. Kennerdell JS, Dekker A, Johnson SL, Dubois PJ. Fine needle aspiration biopsy: its use in orbital tumors. Arch Ophthalmol 1979;97:1315-7. doi:10.1001/archopht.1979.01020020057013. 